\title{
Increased serum concentrations of tissue inhibitor of metalloproteinase-1 in COPD patients
}

\author{
Y. Higashimoto, Y. Yamagata, T. Iwata, M. Okada, T. Ishiguchi, H. Sato, \\ M. Masuda and H. Itoh
}

ABSTRACT: Matrix metalloproteinase (MMP)-9 and tissue inhibitors of metalloproteinase (TIMP)1 concentrations are increased in the sputum of asthma and chronic bronchitis patients, and are thought to be related to airflow obstruction. However, serum concentrations of these enzymes have not been clearly evaluated in patients with chronic obstructive pulmonary disease (COPD). The aim of this study was to examine the serum concentrations of these enzymes in COPD and asthmatic patients in order to determine their relationship with airway obstruction.

Serum samples were obtained from 72 patients with COPD: 66 control subjects and 26 patients with asthma. Smoking histories of control subjects were matched with those of COPD patients. Serum concentrations of TIMP-1 and MMP-9 were determined by ELISA.

The circulating TIMP-1 concentration was significantly higher in stable COPD patients than in control and asthmatic subjects, and was significantly negatively correlated with forced expiratory volume in one second/forced vital capacity in COPD patients. The molar ratio between MMP-9 and TIMP-1 was significantly lower in COPD patients than in control subjects. In patients with COPD, the serum TIMP-1 concentration was significantly increased during disease exacerbation.

In conclusion, the current findings suggest that serum tissue inhibitors of metalloproteinase-1 concentration can be used as a serum marker of airway obstruction and exacerbation in chronic obstructive pulmonary disease patients.

KEYWORDS: Bronchial asthma, chronic obstructive pulmonary disease, exacerbation, metalloproteinases, serum markers, tissue inhibitors of matrix metalloproteinases

$\mathbf{P}$ ulmonary emphysema, the major contributor to morbidity and mortality in patients with chronic obstructive pulmonary disease (COPD), is characterised by progressive destruction of the extracellular matrix (ECM) of the lung [1-4]. The most widely accepted theory of the pathogenesis of pulmonary emphysema is a proteinase-antiproteinase imbalance. Although a number of studies have demonstrated the important role of increased elastolytic activity derived from neutrophils in emphysema $[4,5]$, recent studies have shown that matrix metalloproteinases (MMPs) play key roles in tissue remodelling of the airways in COPD and asthma patients $[2,6-8]$. MMPs are a family of structurally related enzymes that are capable of degrading all components of the ECM [9]. Members of the MMP family are selectively inhibited by tissue inhibitors of metalloproteinases (TIMPs). TIMP-1 has been shown to bind to both the active and precursor form of MMP-9, in a 1:1 proportion, and to inhibit its enzymatic activity. Sputum concentrations of MMP-9 and TIMP-1 are increased in patients with COPD and asthma [2, 10]. RUSSELL et al. [11] showed that alveolar macrophages from patients with COPD release larger amounts of MMP-9 with greater enzymatic activity than those from healthy smokers. Two recent studies found that the MMP-9/TIMP-1 ratio was correlated with the degree of airway obstruction. BosSE et al. [12] reported that the serum MMP-9/TIMP-1 ratio is correlated with steroid responsiveness in moderate-to-severe asthma. In their study, a low MMP-9/TIMP-1 ratio was observed in asthmatic subjects with a poor response to steroid therapy. The molar ratio of MMP-9 to TIMP-1 is also lower in the sputum of asthmatics and patients with chronic bronchitis than in that of control subjects, and this ratio is positively correlated with the forced expiratory volume in one second (FEV1) [8]. These reports suggest that excess amounts of TIMP-1 compared with those of MMP-9 may be related to airway narrowing,
AFFILIATIONS

Dept of Internal Medicine, Wakayama Medical University Kihoku Hospital, Ito-gun, Wakayama Prefecture, Japan.

CORRESPONDENCE

Y. Higashimoto

Wakayama Medical University Kihoku Hospital

219 Myoji

Katsuragi-cho

Ito-gun

Wakayama Prefecture 649-7113

Japan

Fax: 81736222579

E-mail: yhigashi@wakayama-

med.ac.jp

Received:

August 072004

Accepted after revision:

December 132004

SUPPORT STATEMENT

This work was supported by a Grant-in-Aid for Scientific Research (No. 15590822) from the Ministry of Education, Science and Culture of Japan, Tokyo, Japan. 
resulting from remodelling and thickening of the airway wall in asthma and COPD. However, the relationship between circulating concentrations of MMP-9 and TIMP-1 and airway obstruction in patients with COPD has not yet been reported.

In this study, the current authors examined circulating TIMP-1 and MMP-9 concentrations in the serum of patients with COPD, control subjects and asthmatic patients, in order to determine the relationship between these concentrations and airway obstruction.

\section{SUBJECTS AND METHODS \\ Subjects}

In total, 72 patients with COPD, 26 patients with asthma and 66 control patients without airway obstruction were selected for the study from patients seen in the Dept of Internal Medicine of Wakayama Medical University Kihoku Hospital (Ito-gun, Japan). COPD was defined according to the Global initiative for chronic Obstructive Lung Disease (GOLD) criteria [13]. COPD was diagnosed as a post-bronchodilator FEV1 $<80 \%$ of the predicted values and an FEV1/forced vital capacity (FVC) ratio $<70 \%$. Bronchial asthma was diagnosed according to the American Thoracic Society guidelines [14]. None of the control subjects had ever suffered from asthma or chronic respiratory symptoms, and all had FEV1/FVC ratios $>70 \%$. The study was approved by the ethics committee of Wakayama Medical University, and all patients gave informed consent for participation. An exacerbation was defined as the presence, for at least 2 consecutive days, of an increase in any two of the following respiratory symptoms: dyspnoea, sputum purulence, sputum amount, wheezing, cough, and symptoms of the common cold, according to the criteria of SEEMUNGAL et al. [15], ANTHONISEN et al. [16] and RODRIGUEZ-ROISIN [17].

\section{Measurement of TIMP-1 and MMP-9}

Detection of serum total TIMP-1 and MMP-9 was performed using the Biotrak ELISA-based detection system (Amersham Biosciences, Little Chalfont, UK). MMP-9 enzyme activities were measured by MMP-9 Biotrak activity assay system (Amersham Biosciences). All assays were performed following the manufacturer's recommendations.

\section{Visual HRCT score}

Pulmonary emphysema was visually assessed by an expert chest radiologist, unaware of the clinical and lung function data, as described previously [18-20]. On three high-resolution computed tomography (HRCT) slices (at the level of the carina, $5 \mathrm{~cm}$ above and $5 \mathrm{~cm}$ below the carina), the lung parenchyma was assessed for two aspects of emphysema: severity and extent. The three levels were graded and scored separately for the left and right lung, giving a total of six lung fields. The extent of emphysema using the direct observation method was scored on a four-point scale. The extent score was 1 if $<25 \%$ of the lung field was involved; 2 if there was $25-50 \%$ involvement; 3 if $50-75 \%$ involvement; and 4 if $>75 \%$ involvement [20]. Severity was graded on a four-point scale: 0, no emphysema; 1, low HRCT attenuation areas $<5 \mathrm{~mm}$ in diameter with or without vascular pruning; 2, circumscribed low HRCT attenuation areas $>5 \mathrm{~mm}$ in diameter, in addition to those $<5 \mathrm{~mm}$ in diameter (vascular pruning is present, but with normal lung intervening); and 3, diffuse low-attenuation areas without intervening normal lung, or confluent larger low-attenuation areas with vascular pruning and distortion of the branching pattern of the lung, occupying all or almost all of the involved parenchyma. For each of the six lung fields, the extent score was multiplied by that for severity to give a degree of emphysema score. The sum of the product of severity score and extent score for the six lung fields has a potential maximum value of $72[18,19]$.

\section{Statistical analysis}

Results are shown as mean \pm SEM. The differences between patient groups were statistically examined using ANOVA, followed by Fisher's protected least-significant difference (PLSD) test. Comparison of COPD patients with long-term oxygen therapy (LTOT) and those without LTOT was performed using an unpaired t-test. Comparisons of values for the same individual were performed using a paired t-test. Correlation analysis was performed using linear regression and Spearman's nonparametric correlation test; p-values $<0.05$ were considered statistically significant.

\section{RESULTS}

Characteristics of the study patients with COPD, asthmatic patients and control subjects are shown in table 1 . The mean ages of patients with asthma and control subjects were similar, but COPD patients were significantly older than the subjects in the two other groups $(p<0.001)$. Smoking histories (Brinkman's index: the number of cigarettes per day $\times$ the number of years) were similar for control subjects and COPD patients, but the number of Brinkman's index for asthmatic subjects was significantly lower than both control subjects and COPD patients $(\mathrm{p}<0.05)$. In total, 22 COPD patients had chronic respiratory failure (arterial oxygen tension $\left(\mathrm{Pa}_{\mathrm{a}} \mathrm{O}_{2}\right)$ $<7.3 \mathrm{kPa}$ ) and were undergoing LTOT.

The mean serum TIMP-1 concentration of COPD patients was significantly higher than those of control and asthmatic subjects (control: 119.9 \pm 6.5 ; asthma: 162.1 \pm 13.6 ; COPD: $193.0 \pm 5.3 \mathrm{ng} \cdot \mathrm{mL}^{-1} ; \mathrm{p}<0.0001$ and $\mathrm{p}<0.05 ;$ ANOVA, followed

\begin{tabular}{lccc} 
TABLE 1 & Demographic data for subjects & \\
& Control & Asthma & COPD \\
\hline & & & \\
Subjects n & 66 & 26 & 72 \\
Age yrs & $61.6 \pm 1.5$ & $64.6 \pm 4.0^{*}$ & $73.2 \pm 0.7^{\#}$ \\
Males & 66 & 8 & 72 \\
Current smoker & 25 & 4 & 12 \\
Brinkman's index & $1024.7 \pm 79.3$ & $491.6 \pm 205.8^{*}$ & $1368.5 \pm 101.3$ \\
FEV 1 FVC ratio & $0.80 \pm 0.01$ & $0.71 \pm 0.03^{*}$ & $0.56 \pm 0.01^{\#}$ \\
FEV $\mathbf{1}$ L & $2.92 \pm 0.09$ & $1.93 \pm 0.25^{\#}$ & $1.44 \pm 0.07^{\#}$ \\
FEV $\mathbf{1} \%$ pred & $94.5 \pm 1.9$ & $68.1 \pm 4.2^{\#}$ & $55.85 \pm 2.5^{\#}$ \\
FVC L & $3.65 \pm 0.10$ & $2.68 \pm 0.24^{\#}$ & $2.51 \pm 0.09^{\#}$ \\
LTOT & 0 & 0 & 22 \\
\hline
\end{tabular}

Data are presented as mean \pm SEM, unless otherwise stated. COPD: chronic obstructive pulmonary disease; Brinkman's index: the number of cigarettes per day $\times$ the number of years; FEV1: forced expiratory volume in one second; FVC: forced vital capacity; \% pred: \% predicted; LTOT: Iong-term oxygen therapy. " at blood sampling. *: $p<0.05$; \#: $p<0.0001$ (ANOVA, followed by Fisher's protected least-significant difference test) 
by Fisher's PLSD test; fig. 1a). Serum TIMP-1 concentrations of asthmatic patients were also significantly higher than those of the control subjects $(p<0.001$; fig. $1 a)$. The serum TIMP-1 concentration significantly correlated with the FEV1/FVC \% in COPD patients only $(\mathrm{p}<0.05$; Spearman's rank correlation test; fig. 1b).

Blood samples were also obtained from COPD patients when they presented at the hospital with exacerbations. TIMP-1 concentration was increased during exacerbation in nine COPD patients $(\mathrm{p}<0.05$; paired $\mathrm{t}$-test; fig. 2$)$. These COPD patients were treated with a combination of systemic steroids, bronchodilators, theophylline or antibiotics, according to the GOLD criteria [13]. In four of these COPD patients, blood samples were also taken 7-15 days after the exacerbation, in addition to those taken during the exacerbation. Serum TIMP-1 concentrations returned to the pre-exacerbation levels in three out of four patients within 7 days after the exacerbation, but a high level was sustained, even after 15 days, in one patient. An additional five patients had blood samples taken only preexacerbation and during exacerbation.
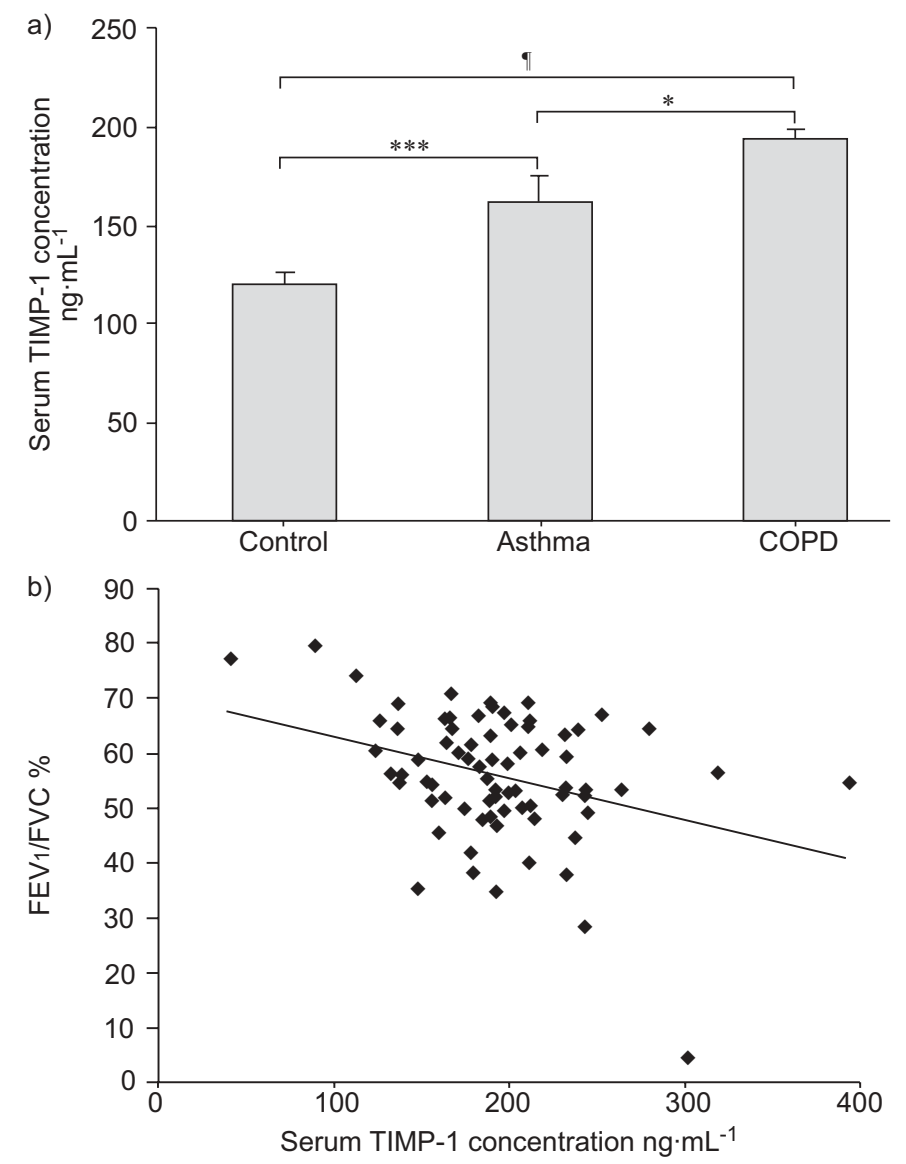

FIGURE 1. a) Serum tissue inhibitors of metalloproteinase (TIMP)-1 concentration in control subjects $(n=66)$, asthmatic subjects $(n=26)$ and patients with chronic obstructive pulmonary disease (COPD; $n=72$ ). ${ }^{*}: p<0.05 ;{ }^{*} * *: p<0.001 ;{ }^{\natural}$ : $p<0.0001$ (ANOVA, followed by Fisher's protected least-significant difference test). b) Correlation between serum TIMP-1 concentration and forced expiratory volume in one second (FEV 1$) /$ forced vital capacity (FVC) \% in COPD patients ( $n=72$; Spearman's rank correlation test; $\mathrm{p}<0.05)$.

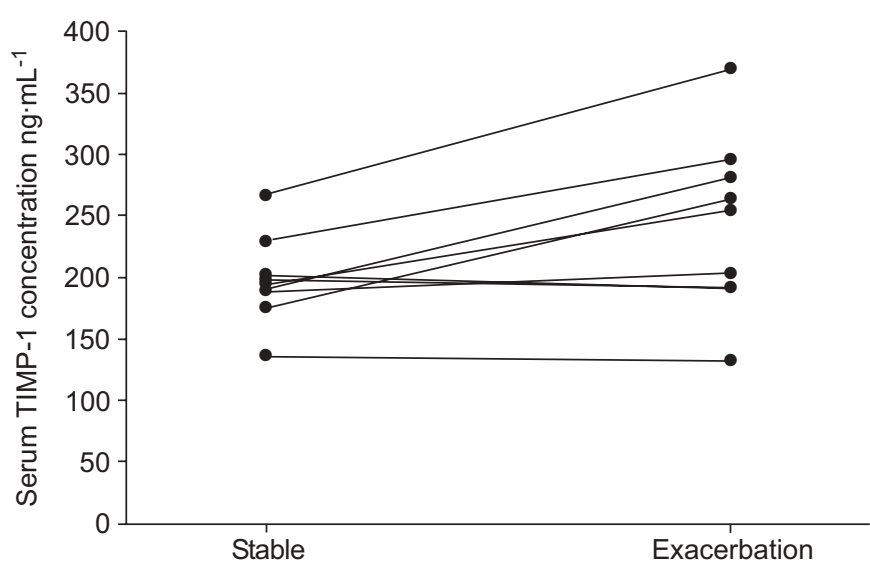

FIGURE 2. Serum tissue inhibitor of metalloproteinase (TIMP)-1 concentration in patients with chronic obstructive pulmonary disease (COPD; paired t-test). Exacerbation: during COPD exacerbation; stable: pre-exacerbation or improvement of symptoms $(p<0.05 ; n=9)$.

Serum MMP-9 concentration did not differ among the three groups (control: 249.2 \pm 19 ; asthma: 248.9 \pm 30 ; COPD: $\left.241.4 \pm 18 \mathrm{ng} \cdot \mathrm{mL}^{-1}\right)$. The serum MMP-9/TIMP-1 molar ratio was significantly lower in patients with COPD than in control subjects (control: $0.57 \pm 0.06$; asthma: 0.44 \pm 0.09 ; COPD: $0.29 \pm 0.02 ; p<0.0001$; fig. 3a). Serum MMP-9 activity correlated well with the protein concentrations $(p<0.0001$; Spearman's rank correlation test; fig. $3 b$ ), and did not differ among the three groups.

Visual emphysema scores were assessed in 67 of the COPD patients who underwent HRCT. Most of the COPD patients had low attenuation areas (visual scores: $18.3 \pm 2.1$; range: 0 $66)$, except nine patients who did not have any low attenuation area in HRCT (score 0). The emphysema scores did not correlate with serum TIMP-1 or MMP-9 levels, but were negatively correlated with FEV1 values $(\mathrm{p}<0.01$; Spearman's rank correlation test).

Serum MMP-9 concentrations of COPD patients undergoing LTOT $\left(293.8 \pm 42.5 \mathrm{ng} \cdot \mathrm{mL}^{-1} ; \mathrm{n}=22\right)$ were significantly higher than those of COPD patients not undergoing LTOT $\left(214.9 \pm 17.7 \mathrm{ng} \cdot \mathrm{mL}^{-1} ; \quad \mathrm{n}=49 ; \quad \mathrm{p}<0.05, \quad\right.$ unpaired $\mathrm{t}$-test $)$. However, serum MMP-9 activities were not different between these two groups, and there was no significant correlation between MMP-9 and FEV1, or MMP-9 and $\mathrm{Pa}_{2} \mathrm{O}_{2}$ at blood sampling.

Nineteen out of 26 asthmatic patients were treated with steroids (inhaled steroids or oral steroids), but this treatment did not influence serum TIMP-1 levels (steroid treatment group: $154.9 \pm 17.4$; nonsteroid group: $181.7 \pm 18.6 \mathrm{ng} \cdot \mathrm{mL}^{-1}$ ) or MMP-9 levels (steroid treatment group: $260.9 \pm 40.1$; nonsteroid group: $216.5 \pm 37.9 \mathrm{ng} \cdot \mathrm{mL}^{-1}$ ). Similarly, 12 out of 72 COPD patients received steroid treatment that did not influence differences in serum TIMP-1 levels or MMP-9 levels.

\section{DISCUSSION}

In the present study, it was found that the circulating TIMP-1 concentration was significantly higher in stable COPD patients than in control and asthmatic subjects, and 

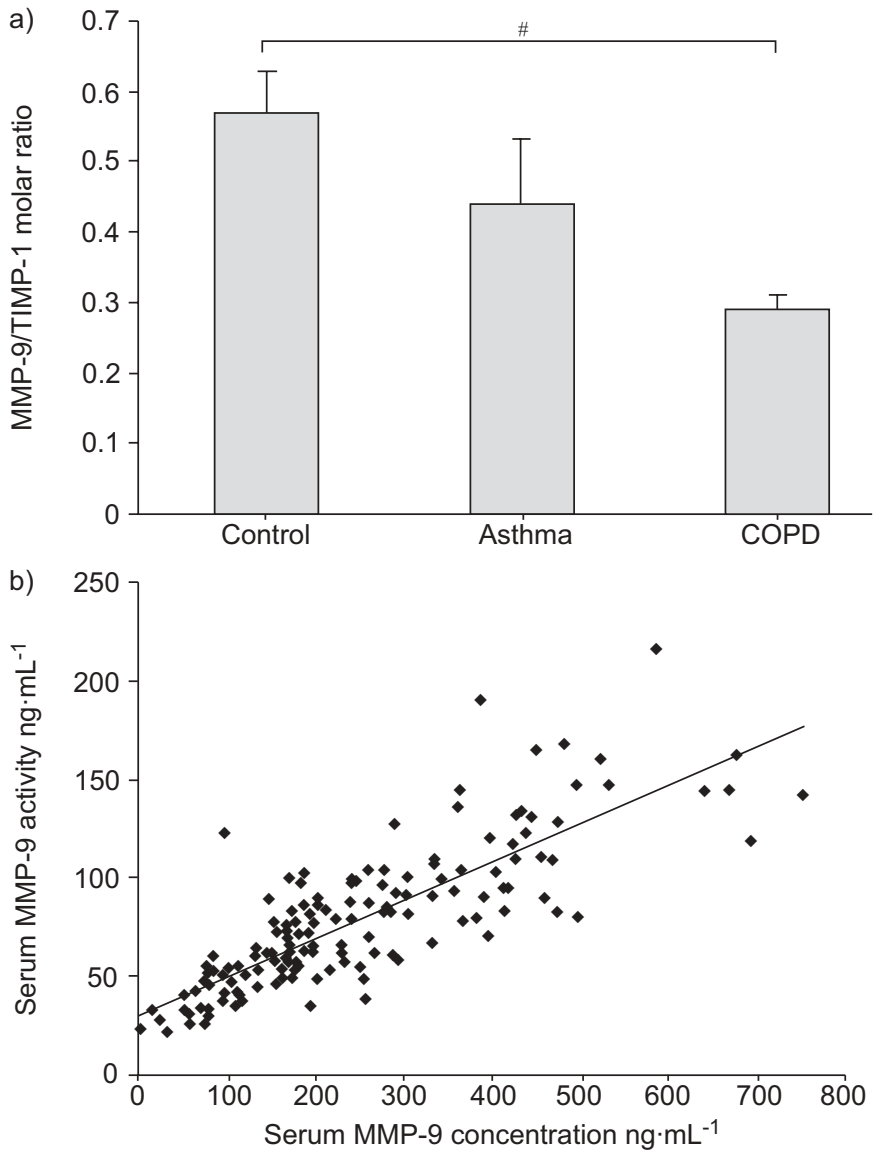

FIGURE 3. a) Molar ratio of matrix metalloproteinase (MMP)-9 and tissue inhibitor of metalloproteinase (TIMP)- 1 in control subjects $(n=66)$, asthmatic subjects $(\mathrm{n}=26)$ and patients with chronic obstructive pulmonary disease (COPD; $n=72$ ). $\#: p<0.0001$ (ANOVA, followed by Fisher's protected least-significant difference test). b) Correlation between serum concentration and activity of MMP-9 in all study patients ( $n=162$; Spearman's rank correlation test).

that this concentration was significantly correlated with the FEV1/FVC \% in COPD patients. To the authors' knowledge, this is the first report relating serum TIMP-1 concentration with airway obstruction in COPD patients.

Recent studies have shown the usefulness of induced sputum [8] and exhaled breath condensate [21] for monitoring airway inflammation in COPD patients. Although these methods are noninvasive and useful for examining local inflammation of the airways, they require time and are not suitable for studying large numbers of patients.

Few studies have examined serum markers of airway obstruction in COPD. RiISE et al. [22] reported that the circulating intercellular adhesion molecule (ICAM)-1 concentration was higher in COPD patients than in control subjects, and that serum levels of circulating E-selectin were significantly correlated with FEV1 per cent predicted. Their study suggested activation and recruitment of neutrophils in COPD patients, since E-selectin is the receptor for neutrophil L-selectin, and ICAM-1 is a ligand for leukocyte receptors.
Neutrophils are an important source of MMP-9 and TIMP-1 [23, 24]. CATALDO et al. [24] reported that the spontaneous release of TIMP-1 and MMP-9 secretion by circulating granulocytes was similar in COPD patients, asthmatic subjects and control subjects. Conversely, sputum MMP-9 activity and TIMP-1 levels have been shown to be increased in COPD and asthmatic subjects compared with control subjects $[2,10]$.

In the present study, increased levels of serum TIMP-1 were found in COPD patients compared with those in asthmatic and control subjects. These results suggest that the increase in serum TIMP-1 levels in COPD patients may not be due to increased secretion from circulating neutrophils, but, instead, to increased secretion from cells in the lung.

In the present study, serum MMP-9 concentrations were similar in COPD patients and control subjects. MAO et al. [7] found that MMP-9 concentrations were higher in COPD patients than in nonsmoking control subjects. This discrepancy in findings may be due to differences in smoking status. The current authors used control subjects whose smoking histories matched those of the COPD patients. Control subjects might, therefore, have also had airway inflammation as a result of smoking alone.

In the present study, the serum MMP-9 concentration was significantly higher in patients with severe COPD undergoing LTOT than in COPD patients not undergoing LTOT. Airway inflammation by neutrophils, macrophages and lymphocytes is known to increase as COPD progresses [25, 26]. These findings suggest that severe COPD features more severe airway inflammation than mild COPD, resulting in increased release of MMP-9 into the circulation. However, MMP-9 levels are not related to parameters such as lung function and arterial blood oxygen saturation.

WINKLER et al. [27] showed that MMP-9 levels are markedly elevated in endotracheal aspirates of children with respiratory failure. Therefore, it can be speculated that circulating MMP-9 may be elevated only in very severe disease with respiratory failure, but not in mild-to-severe disease without respiratory failure.

In patients with COPD, the serum TIMP-1 concentration increased significantly during disease exacerbation. Pulmonary function is believed to decline with frequent exacerbations in COPD patients [28]. It is possible that excess amounts of TIMP-1 may be released into the airways during an exacerbation and may result in progressive remodelling of the airway in COPD patients.

The serum MMP-9/TIMP-1 molar ratio was significantly lower in patients with COPD than in control subjects. TIMP-1 binds noncovalently in a 1:1 proportion to MMP-9 and inhibits its enzymatic activity. TIMP-1 has other biological activities as well. It has been shown to induce the proliferation of skin fibroblasts isolated from patients with systemic sclerosis. The fibroblasts release TIMP-1, which may be may be responsible for the autocrinous promotion of fibrogenesis found in this disease [29]. Bosse et al. [12] reported that the serum MMP-9/ TIMP-1 ratio correlated with steroid responsiveness in moderate-to-severe asthma. Therefore, excess TIMP-1, compared with MMP-9, in COPD patients may be related to the fibrogenic tissue-remodelling processes in the airways, similar to that shown in steroid-resistant asthma [12]. 
To clarify whether the severity of emphysematous change correlated with TIMP-1 levels in COPD patients, the current authors assessed HRCT visual scores of these patients. There was no significant correlation between serum TIMP-1 levels and visual scores, suggesting that serum TIMP-1 levels may be related with airway obstruction rather than emphysematous change.

In conclusion, current findings of increased levels of serum tissue inhibitor of metalloproteinase- 1 and decreased matrix metalloproteinase- $9 /$ tissue inhibitor of metalloproteinase-1 ratio support the hypothesis that excess amounts of tissue inhibitors of metalloproteinase- 1 may be associated with the airway remodelling and narrowing that occurs in chronic obstructive pulmonary disease. Further investigations are necessary to clarify specific cell types that may be involved in the production and secretion of circulating tissue inhibitor of metalloproteinase- 1 and matrix metalloproteinase- 9 in chronic obstructive pulmonary disease patients. Longitudinal studies are required to determine whether these enzymes are indeed associated with progressive disease in chronic obstructive pulmonary disease patients.

\section{REFERENCES}

1 Ohnishi K, Takagi M, Kurokawa Y, Satomi S, Konttinen YT. Matrix metalloproteinase-mediated extracellular matrix protein degradation in human pulmonary emphysema. Lab Invest 1998; 78: 1077-1087.

2 Cataldo D, Munaut C, Noel A, et al. MMP-2- and MMP-9linked gelatinolytic activity in the sputum from patients with asthma and chronic obstructive pulmonary disease. Int Arch Allergy Immunol 2000; 123: 259-267.

3 Kang MJ, Oh YM, Lee JC, et al. Lung matrix metalloproteinase-9 correlates with cigarette smoking and obstruction of airflow. J Korean Med Sci 2003; 18: 821-827.

4 Fujita J, Nelson NL, Daughton DM, et al. Evaluation of elastase and antielastase balance in patients with chronic bronchitis and pulmonary emphysema. Am Rev Respir Dis 1990; 142: 57-62.

5 Ogushi F, Hubbard RC, Vogelmeier C, Fells GA, Crystal RG. Risk factors for emphysema: cigarette smoking is associated with a reduction in the association rate constant of lung alpha 1-antitrypsin for neutrophil elastase. J Clin Invest 1991; 87: 1060-1065.

6 Montano M, Beccerril C, Ruiz V, Ramos C, Sansores RH, Gonzalez-Avila G. Matrix metalloproteinases activity in COPD associated with wood smoke. Chest 2004; 125: 466-472.

7 Mao JT, Tashkin DP, Belloni PN, Baileyhealy I, Baratelli F, Roth MD. All-trans retinoic acid modulates the balance of matrix metalloproteinase-9 and tissue inhibitor of metalloproteinase-1 in patients with emphysema. Chest 2003; 124: 1724-1732.

8 Vignola AM, Riccobono L, Mirabella A, et al. Sputum metalloproteinase-9/tissue inhibitor of metalloproteinase1 ratio correlates with airflow obstruction in asthma and chronic bronchitis. Am J Respir Crit Care Med 1998; 158: 1945-1950.

9 Woessner JF Jr. Matrix metalloproteinases and their inhibitors in connective tissue remodeling. FASEB J 1991; 5: 2145-2154.
10 Beeh KM, Beier J, Kornmann O, Buhl R. Sputum matrix metalloproteinase-9, tissue inhibitor of metalloprotinease1 , and their molar ratio in patients with chronic obstructive pulmonary disease, idiopathic pulmonary fibrosis and healthy subjects. Respir Med 2003; 97: 634-639.

11 Russell RE, Culpitt SV, DeMatos C, et al. Release and activity of matrix metalloproteinase- 9 and tissue inhibitor of metalloproteinase-1 by alveolar macrophages from patients with chronic obstructive pulmonary disease. Am J Respir Cell Mol Biol 2002; 26: 602-609.

12 Bosse M, Chakir J, Rouabhia M, Boulet LP, Audette M, Laviolette M. Serum matrix metalloproteinase-9: tissue inhibitor of metalloproteinase-1 ratio correlates with steroid responsiveness in moderate to severe asthma. Am J Respir Crit Care Med 1999; 159: 596-602.

13 Pauwels RA, Buist AS, Calverley PM, Jenkins CR, Hurd SS. Global strategy for the diagnosis, management, and prevention of chronic obstructive pulmonary disease: NHLBI/WHO Global Initiative for Chronic Obstructive Lung Disease (GOLD) Workshop summary. Am J Respir Crit Care Med 2001; 163: 1256-1276.

14 Standards for the diagnosis and care of patients with chronic obstructive pulmonary disease (COPD) and asthma. Am Rev Respir Dis 1987; 136: 225-244.

15 Seemungal TA, Donaldson GC, Bhowmik A, Jeffries DJ, Wedzicha JA. Time course and recovery of exacerbations in patients with chronic obstructive pulmonary disease. Am J Respir Crit Care Med 2000; 161: 1608-1613.

16 Anthonisen NR, Manfreda J, Warren CP, Hershfield ES, Harding GK, Nelson NA. Antibiotic therapy in exacerbations of chronic obstructive pulmonary disease. Ann Intern Med 1987; 106: 196-204.

17 Rodriguez-Roisin R. Toward a consensus definition for COPD exacerbations. Chest 2000; 117: Suppl. 2, 398S-401S.

18 Sakai F, Gamsu G, Im JG, Ray CS. Pulmonary function abnormalities in patients with CT-determined emphysema. J Comput Assist Tomogr 1987; 11: 963-968.

19 Kohlhaufl M, Brand P, Rock C, et al. Noninvasive diagnosis of emphysema: aerosol morphometry and aerosol bolus dispersion in comparison to HRCT. Am J Respir Crit Care Med 1999; 160: 913-918.

20 Goddard PR, Nicholson EM, Laszlo G, Watt I. Computed tomography in pulmonary emphysema. Clin Radiol 1982; 33: 379-387.

21 Montuschi P, Kharitonov SA, Ciabattoni G, Barnes PJ. Exhaled leukotrienes and prostaglandins in COPD. Thorax 2003; 58: 585-588.

22 Riise GC, Larsson S, Lofdahl CG, Andersson BA. Circulating cell adhesion molecules in bronchial lavage and serum in COPD patients with chronic bronchitis. Eur Respir J 1994; 7: 1673-1677.

23 Cataldo DD, Gueders MM, Rocks N, et al. Pathogenic role of matrix metalloproteases and their inhibitors in asthma and chronic obstructive pulmonary disease and therapeutic relevance of matrix metalloproteases inhibitors. Cell Mol Biol (Noisy-le-grand) 2003; 49: 875-884.

24 Cataldo D, Munaut C, Noel A, et al. Matrix metalloproteinases and TIMP-1 production by peripheral blood granulocytes from COPD patients and asthmatics. Allergy 2001; 56: 145-151. 
25 Hogg JC, Chu F, Utokaparch S, et al. The nature of small-airway obstruction in chronic obstructive pulmonary disease. $N$ Engl J Med 2004; 350: 2645-2653.

26 Retamales I, Elliott WM, Meshi B, et al. Amplification of inflammation in emphysema and its association with latent adenoviral infection. Am J Respir Crit Care Med 2001; 164: 469-473.

27 Winkler MK, Foldes JK, Bunn RC, Fowlkes JL. Implications for matrix metalloproteinases as modulators of pediatric lung disease. Am J Physiol Lung Cell Mol Physiol 2003; 284: L557-L565.

28 Donaldson GC, Seemungal TA, Bhowmik A, Wedzicha JA. Relationship between exacerbation frequency and lung function decline in chronic obstructive pulmonary disease. Thorax 2002; 57: 847-852.

29 Kikuchi K, Kadono T, Furue M, Tamaki K. Tissue inhibitor of metalloproteinase 1 (TIMP-1) may be an autocrine growth factor in scleroderma fibroblasts. I Invest Dermatol 1997; 108: 281-284. 